\title{
Un service d'aide aux étudiants offert par des enseignants experts : la réussite de l'étudiant au cœur des préoccupations
}

\author{
Aid Service Provided by Expert Teachers: Student Success at the Heart \\ of Concerns
}

Sophie LAFLAMME ${ }^{1}$

1 Faculté de médecine et des sciences de la santé, Université de Sherbrooke, Québec, Canada

Manuscrit reçu le 29 octobre 2013 ; commentaires éditoriaux formulés aux auteurs le 28 janvier 2014 ; accepté pour publication le 22 février 2014

\section{Mots-clés \\ Faculté de médecine et des sciences de la santé ; service d'aide ; conseils académiques ; enseignant expert ; succès des étudiants}

\section{Keywords}

Faculty of medicine and health sciences; aid service; academic support; expert teacher; student success
Résumé - Contexte : Les services de conseil académique sont fondamentaux aux programmes de médecine et des sciences de la santé car les étudiants subissent un niveau de stress important pouvant se répercuter sur la performance académique et conduire à l'abandon des études. Objectifs : Cet article rapporte l'expérience du service d'adjoints à la vie étudiante de la Faculté de médecine et des sciences de la santé (FMSS) de l'Université de Sherbrooke. Description et bilan d'activité du dispositif : Le service est formé de 22 adjoints qui offrent une aide bienveillante et confidentielle à l'ensemble des étudiants de la FMSS qui en font la demande. Il est encadré par un coordonnateur ainsi qu'une vice-doyenne et vise à : 1) rendre disponible un service structuré et de qualité offert par du personnel compétent ;2) favoriser la continuité et l'accessibilité du service aux étudiants et : 3) offrir une réponse adaptée à leurs besoins au plan personnel, académique et de leur développement professionnel. Discussion et conclusion : Le service connaît une popularité croissante chez les étudiants. Il réunit des enseignants de plusieurs programmes d'études bénéficiant d'encadrement, de formations continues et évoluant en groupe, ce qui favorise le réseautage et le partage des expériences. Ces enseignants qui ont eux-mêmes étudié dans les programmes d'études de la faculté sont en mesure de partager avec les étudiants les expériences et difficultés qu'ils ont vécues. Les sept années d'expérimentation de ce service ont convaincu la FMSS de sa pertinence ainsi que de sa contribution réelle au bien-être et au développement des étudiants.

Abstract - Background: Academic council services are fundamental in medicine and health sciences programs where students are exposed to considerable stress, which can impact their academic performance and lead to dropping out. Objective: The paper presents a unique aid service, the student life assistant service, available at the faculty of medicine and health sciences (Faculté de médecine et des sciences de la santé - FMSS) of Université de Sherbrooke. Program description: The service consists of 22 faculty teachers who provide considerate and confidential help to all FMSS students who request it. Managed by a coordinator and an assistant dean it is designed to: 1) offer structured quality service from experienced teachers, 
2) promote continuity and accessibility of service to students and 3) provide answers suited to student needs in terms of personal, academic and professional development. Discussion and conclusion: The service is increasingly popular among students. It brings together teachers from various FMSS academic programs who provide coaching, ongoing training and group progress tutoring, all of which fosters networking and experience sharing. Since the teachers are former students in the FMSS programs, they are able to share the experiences and difficulties they themselves went through. After 7 years spent on improving the service the faculty was convinced of its relevance and tangible contribution to student well-being and development.

\section{Problématique}

Le stress et la détresse : une préoccupation importante chez les étudiants de médecine et des sciences de la santé

Le nombre d'étudiants universitaires aux prises avec des problèmes de détresse émotionnelle et d'anxiété a considérablement augmenté dans les dernières années $^{[1-3]}$. Ces problèmes affectent particulièrement les étudiants en médecine et en sciences de la santé, qui sont soumis à un niveau de stress important durant leurs études ${ }^{[4]}$. Ce stress est notamment attribuable à des impératifs de performance, à une charge de travail importante, à des difficultés financières, aux exigences de la pratique clinique et, pour certains, à des problématiques familiales ou personnelles ${ }^{[5,6]}$. En outre, des taux de dépression et d'anxiété de l'ordre de $25 \%$ à $56 \%$ ont été rapportés chez des étudiants américains et canadiens inscrits au programme de doctorat en médecine, surpassant les taux habituellement observés dans la population général $^{[5]}$. Des niveaux de stress et de détresse émotionnelle significatifs ont aussi été rapportés chez des étudiants de physiothérapie, de sciences infirmières, de pharmacie et ce, tant au niveau du baccalauréat (licence dans certains pays francophones) qu'à des niveaux supérieurs ${ }^{[4,7,8]}$. La situation est d'autant plus préoccupante que ces étudiants n'ont généralement pas tendance à rechercher de l'aide lorsqu'ils en ont besoin ${ }^{[12]}$, certains percevant la demande d'aide comme un signe de faiblesse ou craignant plutôt de compromettre leur vie professionnelle ${ }^{[5,9]}$. Par ailleurs, certains étudiants ne seraient pas convaincus de pouvoir être bien compris ou auraient peur d'être stigmatisés $^{[2,9]}$.

\section{Mieux soutenir les étudiants grâce à des modalités} concrètes

Les établissements d'enseignement ont pris la mesure de ces difficultés et ont progressivement mis en place différents services qui sont offerts à l'ensemble de leurs étudiants, parmi lesquels des services de soutien administratif, psychologique, pédagogique, d'orientation, des services médicaux ou encore financiers. Ces services sont reconnus pour jouer un rôle significatif dans le développement de l'étudiant et dans son succès académique ${ }^{[10]}$. Des services facultaires se sont par ailleurs progressivement ajoutés à l'offre plus traditionnelle des services offerts à grande échelle par les établissements. Plus spécifiquement, certaines facultés offrent une panoplie de services de soutien à leurs étudiants, qu'il s'agisse de soutien psychologique professionnel, de counseling pédagogique ou académique, d'un système de pairs aidants (groupe d'étudiants qui assistent des étudiants, comme, par exemple, le Centre de soutien aux étudiants de l'Université Laval) ou de parrainage (étudiants plus anciens assignés à de nouveaux étudiants, comme, par exemple, le Programme de parrainage de l'Université d'Ottawa). Il existe aussi des programmes de mentorats individuels, des programmes ciblés de soutien au bien-être moral et à la santé des étudiants (comme, par exemple, The Vanderbilt Medical Student Wellness Program) ou encore, des programmes de gestion du stress $^{[6,11-13]}$.

Chacune de ces initiatives peut être créditée d'une certaine efficacité dans la mesure où elle parvient à bien répondre aux besoins des étudiants, des enseignants et autres membres du personnel facultaire impliqués, et de l'institution elle-même ${ }^{[14]}$. À cet égard, certaines conditions de succès des services de conseil académique ont été identifiées ; 
elles ont trait aux qualités du contenu et des interactions avec les étudiants, à la disponibilité de mécanismes de renforcement et de développement professionnel pour ceux qui prodiguent les services, à l'existence d'un soutien au plan structurel et organisationnel (par exemple en termes de ressources humaines et financières, de leadership), ou au plan des politiques internes (par exemple, la mission de l'établissement) ${ }^{[13-15]}$.

Le service d'adjoints à la vie étudiante de la Faculté de médecine et des sciences de la santé (FMSS) de l'Université de Sherbrooke : un investissement pour le bien-être des étudiants

Soucieuse du bien-être de ses étudiants ainsi que de leur succès académique, la FMSS de l'Université de Sherbrooke a choisi d'investir dans le bien-être émotionnel de ses étudiants en instituant un service d'adjoints à la vie étudiante. Offert par des enseignants experts, le service est chargé d'apporter aux étudiants de la faculté une aide et un soutien, axés sur leurs besoins. Après sept années d'expérimentation, la FMSS est convaincue de la pertinence de ce dispositif et de sa contribution essentielle au bien-être et au développement personnel et professionnel de ses étudiants.

À notre connaissance, aucune expérience de ce type de service dans les facultés des disciplines de santé n'est actuellement documentée dans la littérature francophone et anglophone disponible. L'objectif de la présente contribution est ainsi de rapporter l'expérience du service d'adjoints à la vie étudiante de FMSS.

\section{Description et bilan d'activité du dispositif}

La mise sur pied du service d'adjoints à la vie étudiante repose sur les objectifs suivants, lesquels ont guidé l'ensemble de sa démarche de création et d'implantation : 1) rendre disponible un service structuré et de qualité offert par du personnel compétent ;
2) favoriser la continuité et l'accessibilité du service aux étudiants ; 3) offrir une réponse adaptée aux besoins des étudiants aux plans personnel, académique et de leur développement professionnel.

Un service structuré et de qualité offert par du personnel compétent

\section{Une structure en réseau}

Le service d'adjoints à la vie étudiante est enchâssé dans une structure organisée et bien établie. Il a d'abord été déployé dans le cadre du programme de doctorat en médecine, en réponse aux besoins exprimés par des étudiants qui rencontraient des difficultés importantes durant leur parcours d'études et qui acceptaient d'en parler avec leurs enseignants mais n'osaient pas consulter. Le service s'est par la suite élargi à l'ensemble des autres programmes de la faculté, après que les administrateurs aient constaté des besoins similaires chez les autres étudiants. Il est aujourd'hui disponible pour tous les étudiants des programmes de la faculté sur le campus de Sherbrooke et dans ses sites délocalisés (Saguenay, Moncton, Charles-Lemoyne), soit ceux du doctorat en médecine, des sciences infirmières, de réadaptation, de pharmacologie, de biochimie de la santé et des études supérieures. Les adjoints à la vie étudiante constituent un réseau de 22 enseignants issus de l'ensemble de ces programmes (Fig. 1).

\section{Une coordination soutenante et des orientations claires}

Les adjoints relèvent d'un coordonnateur ainsi que de la vice-doyenne à la vie étudiante et secrétaire de la FMSS (Fig. 1). Le mandat du coordonnateur dure en moyenne de trois à cinq ans et consiste à gérer l'équipe d'adjoints, à prendre en charge leur recrutement, leur encadrement et leur soutien, et à organiser des réunions et des formations. La vice-doyenne se charge de formuler annuellement les grandes lignes directrices et les orientations stratégiques du service, et travaille en collaboration étroite avec le coordonnateur. 


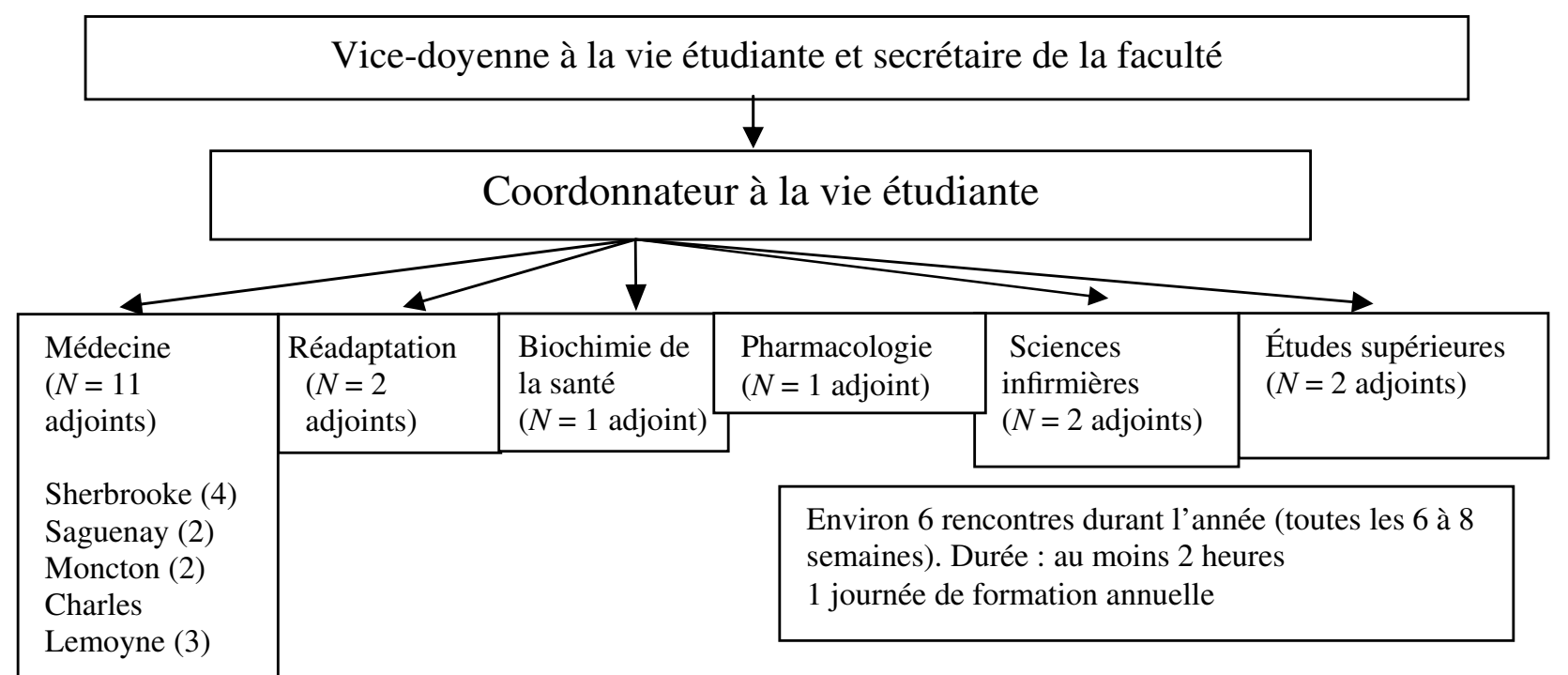

Fig. 1. Structure du service - Adjoints à la vie étudiante.

\section{Une formation et un encadrement continus des adjoints}

Lors de leur assignation, les adjoints sont orientés par le coordonnateur du service et sont informés de l'ensemble des ressources universitaires et communautaires existantes, susceptibles de venir en aide aux étudiants. Ultérieurement, ils peuvent contacter au besoin le coordonnateur pour être guidés plus spécifiquement dans leur rôle. Les adjoints à la vie étudiante se réunissent plusieurs fois par année à des fins de formation continue, de développement et de partage. Ces rencontres leur offrent la possibilité de faire le point sur leur rôle et d'échanger avec leurs collègues sur différentes situations vécues afin de poser un regard critique constructif sur leurs interventions. De plus, les adjoints participent annuellement à une journée de formation sur plusieurs sujets qu'ils ont identifiés durant l'année (par exemple, la dépression, les idées suicidaires, l'abus de substances, le harcèlement et l'intimidation, le cadre légal, la confidentialité, le professionnalisme). Ces séances de formation comportent généralement des présentations effectuées par des experts de contenus et des ateliers de discussion.

\section{Une préoccupation constante de confidentialité}

Les adjoints sont tenus, dans le cadre de leurs fonctions, de préserver la confidentialité de tout ce qui est discuté lors de la consultation et aucun lien ne peut être établi avec le dossier académique de l'étudiant. Le maintien de la confidentialité est souligné dans les publicités du service ou lorsqu'il est présenté aux étudiants. Des ruptures de confidentialité encadrées peuvent toutefois se produire lors de situations extrêmes où un étudiant présente un danger élevé pour luimême (par exemple, dans le cas d'idées suicidaires) ou pour autrui.

\section{Un service continu facilement accessible} aux étudiants

\section{Une promotion continue du service}

Tous les étudiants sont informés de l'existence du service en début d'année lors de l'accueil étudiant et à différents moments stratégiques de leur cheminement d'études. Des rappels courriels sont également 
acheminés à l'ensemble des étudiants à des moments charnières de l'année où le nombre de consultation du service est particulièrement élevé. Enfin, le service est publicisé à travers les Intranet de programmes et le site Internet facultaire.

\section{Une utilisation du service par l'étudiant sur une base volontaire}

Le service est sollicité par l'étudiant sur une base volontaire. Ce dernier prend l'initiative de le consulter ou peut le consulter à la suggestion d'une tierce personne (professeur, directeur de programme) de la faculté. L'adjoint pourra alors soutenir l'étudiant dans son développement personnel, lui fournir l' aide adaptée, l'écouter, le guider, le rassurer, le conseiller ou, au besoin, l'orienter vers d'autres ressources adaptées.

\section{Un contact facile avec le service}

L'étudiant peut consulter l'adjoint de son choix dans la faculté, qu'il soit lié ou non à son programme. La liste complète des adjoints, leurs photos ainsi que leurs coordonnées sont disponibles sur la section vie étudiante du site Internet facultaire. Des correspondances et des échanges téléphoniques peuvent par la suite se solder par un rendez-vous en personne avec l'adjoint, d'une durée d'environ une heure. L'étudiant peut être vu jusqu'à trois reprises par l'adjoint qui, au besoin, peut ensuite rediriger l'étudiant vers un service spécifique universitaire ou communautaire, qui pourra lui offrir un suivi adapté à plus long terme.

Une réponse adaptée aux besoins des étudiants

\section{Un service apte à s'adapter à une diversité de motifs de consultation}

Le service connaît une popularité croissante chez l'ensemble des étudiants de la FMSS. Les statistiques de fréquentation de l'année académique 2011-2012 concernant les programmes de médecine, de réadaptation, de biochimie de la santé et de pharmacologie de la FMSS indiquent en effet que 190 des 2135 étudiants inscrits à l'automne 2011 ont bénéficié du service (tableau I), ce qui représente 267 rencontres individuelles. Ces données sont réunies autour de huit grandes catégories de consultations liées aux difficultés personnelles ou familiales, relationnelles et interpersonnelles, académiques, psychologiques, à des problématiques de santé physique, à des préoccupations de prévention, à des projets personnels et aux conseils à un tiers. L'encadré 1 présente des exemples de situations déjà vécues qui ont amenées des étudiants à consulter.

\section{La popularité du service en statistiques}

Les données recueillies montrent que les statistiques de consultation sont relativement similaires d'un programme à l'autre. Elles révèlent en effet que, dans le cadre des programmes de doctorat en médecine, de réadaptation, de biochimie de la santé et de pharmacologie, les adjoints ont été consultés dans une proportion moyenne de $32 \%$, pour des difficultés académiques, de $28 \%$, pour des difficultés psychologiques et de $26 \%$, pour des difficultés personnelles ou familiales (tableau I). Les consultations liées aux difficultés relationnelles $(8 \%)$ et aux problèmes de santé physique $(3 \%)$ sont moins nombreuses, de même que celles liées à la prévention ( $2 \%)$, à des projets personnels $(0,0025 \%)$ et au conseil à un tiers $(0,0065 \%)$. Des taux contrastés de fréquentation sont cependant observés pour certaines catégories de consultations dans certains programmes. En outre, le programme de biochimie de la santé présente un taux très élevé de consultation pour des difficultés académiques (68\%), alors que le programme de réadaptation a des taux de consultation élevés pour des difficultés psychologiques $(42 \%)$ et celui de pharmacologie, pour les conseils à un tiers $(25 \%)$. Ces taux contrastés peuvent être attribuables à de multiples facteurs. Ainsi, le programme de biochimie de la santé connaît un faible contingentement comparativement aux autres programmes, ce qui peut expliquer des niveaux de performance académique très différents chez les étudiants à leur arrivée, sources de fortes pressions au plan académique chez certains d'entre eux. Les étudiants en réadaptation sont, par ailleurs, beaucoup 
Tableau I. Motifs de consultation d'un adjoint à la vie étudiante par les étudiants de la Faculté de médecine et des sciences de la santé de Sherbrooke.

\begin{tabular}{|c|c|c|c|c|c|}
\hline & $\begin{array}{c}\text { Taux pour } \\
\text { l'ensemble } \\
\text { des programmes } \\
(N=267)\end{array}$ & $\begin{array}{c}\text { Médecine } \\
\text { (prédoc \& postdoc) } \\
(N=144)\end{array}$ & $\begin{array}{l}\text { Réadaptation } \\
\text { (Ergothérapie } \\
\text { et physiothérapie) } \\
\qquad(N=63)\end{array}$ & $\begin{array}{l}\text { Pharmacologie } \\
\qquad(N=13)\end{array}$ & $\begin{array}{l}\text { Biochimie } \\
\text { de la santé } \\
(N=39)\end{array}$ \\
\hline $\begin{array}{l}\text { Difficultés personnelles } \\
\text { ou familiales }\end{array}$ & $26 \%$ & $24 \%$ & $29 \%$ & $30 \%$ & $20 \%$ \\
\hline $\begin{array}{l}\text { Difficultés relations } \\
\text { interpersonnelles }\end{array}$ & $8 \%$ & $6 \%$ & $11 \%$ & $5 \%$ & $8 \%$ \\
\hline Difficultés académiques & $32 \%$ & $31 \%$ & $13 \%$ & $15 \%$ & $68 \%$ \\
\hline $\begin{array}{l}\text { Difficultés } \\
\text { psychologiques }\end{array}$ & $28 \%$ & $30 \%$ & $42 \%$ & $20 \%$ & $2 \%$ \\
\hline $\begin{array}{l}\text { Problèmes de santé } \\
\text { physique }\end{array}$ & $3 \%$ & $5 \%$ & $5 \%$ & $0 \%$ & $2 \%$ \\
\hline Prévention & $2 \%$ & $3 \%$ & $0 \%$ & $5 \%$ & $0 \%$ \\
\hline Projet & $0,0025 \%$ & $1 \%$ & $0 \%$ & $0 \%$ & $0 \%$ \\
\hline Conseil à un tiers & $0,0065 \%$ & $0 \%$ & $0 \%$ & $25 \%$ & $0 \%$ \\
\hline
\end{tabular}

* Données fournies par le coordonnateur du service d'adjoints à la vie étudiante de la Faculté de médecine et des sciences de la santé de l'Université de Sherbrooke.

** Il est à noter que les consultations des programmes en sciences infirmières n'ont pas pu être comptabilisées pour des raisons administratives et qu'on ne dispose d'aucun portrait précis de la situation des programmes d'études supérieures parce que leurs étudiants proviennent d'autres facultés de l'Université de Sherbrooke.

plus susceptibles de vivre une instabilité au plan psychologique comparativement aux étudiants des autres programmes, dans la mesure où certains arrivent directement des études collégiales ou proviennent de régions éloignées de Sherbrooke. La pharmacologie est, pour sa part, un programme à petite échelle. L'adjoint y est donc très présent et détient une excellente légitimité auprès de ses collègues enseignants. Ceux-ci peuvent ainsi être amenés à vouloir davantage obtenir son avis à différents égards, d'où le taux élevé de consultation pour les conseils à un tiers.

\section{Une meilleure connaissance des besoins des étudiants}

Ces données permettent d'identifier, pour chacun des programmes de la FMSS, des défis spécifiques à relever en fonction de leur nature, des ressources en place et des caractéristiques particulières des cohortes d'étudiants. Ce portrait statistique peut également s'avérer utile pour cibler des problématiques plus spécifiques ou criantes, et révéler des besoins et des préoccupations importantes chez les étudiants, qui méritent d'être pris en compte. Le coordonnateur du service a le mandat, avec la collaboration étroite de la vice-doyenne, de faire le suivi de ces données auprès des adjoints et des administrateurs des programmes, pour discuter et assurer l'implantation de moyens structurants permettant d'agir de façon préventive auprès des étudiants face à des problématiques récurrentes ainsi soulevées.

\section{Discussion}

Chaque programme d'études comporte ses propres défis et affecte différemment l'état d'esprit et le moral 


\section{Encadré 1}

\section{Exemples de situations conduisant à une demande de consultation}

Difficultés personnelles ou familiales. Un étudiant consulte un adjoint car il se sent envahi par des émotions négatives suite à une rupture amoureuse et au suicide d'un ami. L'adjoint accueille l'étudiant en crise, l'écoute, le rassure, vérifie qu'il dispose de ressources personnelles suffisantes pour surmonter ses difficultés et le réfère au service de psychologie de l'université. Avec l'accord de l'étudiant, l'adjoint se charge de faire un suivi confidentiel avec le programme d'études concerné pour permettre à l'étudiant de bénéficier d'un temps d'arrêt de ses études.

Difficultés relationnelles et interpersonnelles. Un étudiant souffre de sa relation avec un de ses tuteurs de cours et craint que la rétroaction négative qu'il obtient de sa part ne se répercute sur son résultat scolaire. Il consulte un adjoint qui le conseille sur une approche communicationnelle facilitante pour aborder la problématique avec son tuteur.

Difficultés académiques. Un étudiant se sent débordé par ses études et obtient de mauvais résultats scolaires. Il consulte un adjoint pour être outillé au plan de ses méthodes d'études. L'adjoint pourra, au besoin, le référer à un conseiller pédagogique.

Difficultés psychologiques. Un étudiant consulte un adjoint parce qu'il éprouve de la difficulté à gérer son stress. L'adjoint évalue sommairement le niveau de stress de l'étudiant, cherche à normaliser la situation du mieux qu'il peut et réfère au besoin l'étudiant à un service psychologique spécialisé.

Difficultés reliées à des problématiques de santé physique. Un étudiant s'est cassé une jambe et consulte un adjoint pour mieux s'organiser pendant sa convalescence. L'adjoint évalue sa situation et le réfère à son programme d'études pour prendre des arrangements académiques.

Préoccupations de prévention. Un étudiant constate rapidement une faiblesse de sa part dans sa gestion du temps et du travail. Il a déjà réfléchi à des pistes de solutions qu'il souhaite valider avec un adjoint.

Projets personnels. Un étudiant sollicite le soutien et l'avis d'un adjoint dans le cadre d'un montage de son dossier personnel en vue d'un concours.

Conseils à un tiers. Un adjoint est amené à offrir des conseils à un enseignant quant à la conduite à adopter auprès de certains étudiants.

des étudiants. Au moment même où ils acquièrent de nouveaux savoirs et développent leurs compétences professionnelles, ceux-ci doivent apprendre à reconnaître leurs besoins et à savoir s'accorder un répit lorsque c'est nécessaire. Ils doivent arriver à composer avec le stress important et ses conséquences, qui accompagnent leur parcours d'études ${ }^{[2,3]}$. Certains établissements universitaires se sont déjà interrogés sur ces enjeux et ont mis en place un certain nombre de ressources de soutien destinées aux étudiants en difficulté émotionnelle ou morale, dont des soutiens par les pairs ${ }^{[2,3,5]}$, ou encore des programmes structurés ciblant des problématiques particulières ${ }^{[5]}$. Les initiatives dans ce sens demeurent cependant encore peu documentées ${ }^{[3,4]}$. Cet article a voulu présenter un service d'adjoints à la vie étudiante unique et innovant, offert par des enseignants experts engagés à soutenir les étudiants dans leur développement personnel, académique et professionnel, et à favoriser leur rayonnement afin de les mener vers la réussite. Le succès du service d'adjoints à la vie étudiante auprès des étudiants de la FMSS mérite d'être partagé pour peut-être inspirer, à différents niveaux, d'autres programmes universitaires. 


\section{Quelques points forts}

\section{Des conditions organisationnelles favorisant l'émergence d'initiatives de soutien}

La FMSS se caractérise par une culture où la personne occupe une place prépondérante. Les initiatives visant à soutenir la personne et à promouvoir son développement sont donc encouragées, et leur réalisation est facilitée grâce à une multitude de conditions favorables. D'une part, certains fonds communs permettent de soutenir financièrement la création de ce projet et contribuent à assurer sa continuité. D'autre part, l'implication des professeurs comme adjoints à la vie étudiante est formellement reconnue par l'organisation et rémunérée. Effectivement, cette implication humaine est perçue comme équivalent à d'autres types d'implications associées au rôle de professeur, relevant davantage de l'enseignement, de la pratique clinique ou de la recherche. Ces conditions sont essentielles à la réussite et à l'efficacité de services de conseils académiques $^{[14]}$.

\section{La formation d'une communauté de pratique guidée par des objectifs et intérêts communs}

Une autre force du service réside dans le fait qu'il rassemble plusieurs programmes d'études et réunit des enseignants de différents horizons disciplinaires en santé, qui ont en commun l'intérêt de l'étudiant et qui peuvent communiquer entre eux à des fins de soutien mutuel. Une vaste étude américaine révélait en 2005 que l'amélioration des communications entre conseillers académiques représentait un défi fondamental à relever et les répondants à l'enquête reconnaissaient comme indispensable la mise en place d'activités destiner à favoriser une bonne communication entre eux et à leur permettre de travailler ensemble $^{[13]}$. Ils souhaitaient que les lignes de communications entre conseillers soient renforcées et qu'ils puissent bénéficier de contacts plus systématiques et structurés entre eux ${ }^{[13]}$. Le service d'adjoints permet de répondre à ces enjeux. Le fait d'être un groupe favorise le réseautage, le partage des expériences et permet le développement rapide et solide d'une expertise autour de problématiques données.
La formule en réseau transmet également un message clair aux étudiants et à l'ensemble des professeurs et du personnel de la FMSS, en indiquant que la faculté place au premier plan, le soutien et le développement de ses étudiants de même que l'interdisciplinarité et qu'elle n'hésite pas à y investir des ressources humaines et matérielles, autre élément de grande importance ${ }^{[13]}$. À notre connaissance, le service d'adjoints à la vie étudiante est le premier groupe interdisciplinaire mis en place afin d'offrir un soutien aux étudiants au plan de leur bien-être émotionnel, moral, de leur parcours académique et leur développement professionnel. L'étudiant en tire profit. Il est amené à réfléchir à l'importance de prendre en main son propre bien-être, sa santé et son développement personnel et professionnel, ce qui le prépare pour sa future carrière ${ }^{[16]}$. Il est aussi sensibilisé à l'importance de la collaboration interdisciplinaire.

\section{Le souci constant d'aider les aidants}

Le service d'adjoints s'appuie sur un encadrement et des formations continues. Des activités de formation continue sur mesure sont dispensées aux adjoints dans le cadre de rencontres régulières et de journées annuelles axées sur des thèmes d'intérêts communs. La composante « formation et encadrement » du service apporte une valeur ajoutée particulièrement soulignée dans les écrits ${ }^{[17]}$.

\section{La fréquentation du service comme indice de son succès}

La fréquentation du service par les étudiants de la FMSS est bien établie, probablement grâce à l'assurance de la préservation de la confidentialité des consultations, autre condition centrale pour une demande d'aide réalisée à l'intérieur d'un programme d'études $^{[2,5,9,11]}$. La fréquentation du service peut aussi être attribuée à la formule qui laisse à l'étudiant une grande liberté. Ce dernier a le loisir de consulter l'adjoint de son choix sur la base de certaines affinités. Enfin, le service est offert par des enseignants experts qui ont été eux-mêmes des étudiants dans l'un ou l'autre des programmes d'études de la faculté. Ils sont ainsi en mesure de partager leurs propres expériences 
et difficultés vécues durant leurs études et de partager avec les étudiants les solutions qu'ils ont mises en œuvre pour les surmonter ${ }^{[5]}$. En quelque sorte, ils peuvent agir comme modèles de rôles auprès des étudiants $^{[11,18]}$, ce qui est reconnu pour contribuer au développement personnel, professionnel et académique de l'étudiant ${ }^{[2]}$.

\section{Difficultés et limites}

L'implantation et la pérennisation du service d'adjoints à la vie étudiante a bien entendu comporté certains défis, mais aucune contrainte organisationnelle n'est venue freiner le projet de façon importante. En fait, l'adhésion de tous envers le projet a, dès le départ, été manifeste, ce qui a permis de concrétiser facilement et rapidement le projet. Toutefois, bien que déployé depuis quelques années, le service n'a pas encore fait l'objet d'une évaluation organisationnelle minutieuse. À part certaines statistiques de consultation qui démontrent une croissance constante de la popularité du service auprès des étudiants, la FMSS ne dispose pas à ce jour de données précises reliées à la satisfaction des étudiants utilisateurs quant aux soutiens reçus. Des pas en ce sens seront franchis prochainement par la FMSS afin de disposer d'un portrait plus clair et complet de la satisfaction des étudiants envers le service et des bénéfices qu'ils ont tirés de la consultation de leurs adjoints.

\section{Pistes d'avenir}

Depuis son implantation, le service d'adjoints à la vie étudiante de la FMSS s'est surtout attaché à offrir une aide solide et bien organisée aux étudiants au niveau $\mathrm{du}$ soutien personnel. Ce volet du service est aujourd'hui bien rôdé. Plus récemment, le mandat du service s'est élargi pour s'étendre, en plus, vers le développement professionnel et le rayonnement des étudiants. Ceux-ci ont en effet manifesté l'intérêt d'être soutenus dans leurs projets personnels, leur implication dans la communauté ou d'être aidés pour optimiser leur curriculum (par exemple en participant à des concours, en sollicitant des programmes de bourses ou en développant leur plan de carrière). Les adjoints sont désireux de s'investir à ces niveaux et de mettre à profit leurs expériences et expertises pour soutenir ces étudiants dans la promotion de leur développement professionnel et de leur rayonnement. Quelques étudiants ont déjà sollicité l'aide des adjoints dans le cadre de ces nouveaux mandats. Les adjoints pourront éventuellement piloter eux-mêmes des comités de bénévolat ou des projets à vocation communautaire ou de promotion de la santé. Le but visé est de permettre aux étudiants de la FMSS de se développer professionnellement et de s'impliquer dans des initiatives para scolaires positives.

\section{Conclusion}

Les établissements universitaires ont l'obligation morale d'offrir un milieu sécurisant pour leurs étudiants. Ils doivent se préoccuper de créer un climat de communication ouvert, afin que les étudiants qui se sentent lésés ou qui rencontrent des difficultés se sentent suffisamment en confiance pour demander de l'aide. Ils ont la responsabilité de mettre en place des stratégies permettant d'assurer, de prévenir et de résoudre les difficultés auxquelles ils font face ${ }^{[18]}$. Le service d'adjoints à la vie étudiante de la FMSS permet de bien répondre à ces enjeux. Il se présente comme étant une mesure d'encadrement institutionnel interdisciplinaire, qui mise sur l'aide et le soutien confidentiel des étudiants pour les mener à la réussite. De plus, la mise en place de ce service s'inscrit en cohérence directe avec les exigences relatives au bien-être des étudiants, de plus en plus prescrites aux établissements d'enseignement dans le cadre de démarches d'accréditation, de certification ou d'agrément.

\section{Contribution}

Sophie Laflamme a entièrement conceptualisé et rédigé le manuscrit, et a participé activement au recueil, à l'organisation et la présentation des données. 


\section{Approbation éthique}

Non sollicitée

\section{Déclaration d'intérêts}

SL déclare ne pas avoir de conflit d'intérêt financier en lien avec l'objet de cet article. Elle occupe cependant la position institutionnelle de secrétaire et vicedoyenne à la vie étudiante à la Faculté de médecine et des sciences de la santé de l'Université de Sherbrooke et, à ce titre, est responsable du service dont l'expérience est rapportée.

\section{Références}

1. Benton SA, Robertson JM, Tseng W-C, Newton FB, Benton SL. Changes in Counseling Center Client Programs Across 13 Years. Professional Psychology: Research and Practice 2003;34:66-72.

2. Storrie K, Ahern K, Tuckett A. A Systematic Review: Students with Mental Health Problems - A Growing Problem. Int J Nurs Pract 2010;16:1-6.

3. Warwick I, Maxwell C, Statham J, Aggleton P, Simon A. Supporting Mental Health and Emotional WellBeing among Younger Students in Further Education. Journal of Further and Higher Education 2008; 32:1-13.

4. Tucker B, Jones S, Mandy A. Physiotherapy Students' Sources of Stress, Perceived Course Difficulty, and Paid Employment: Comparison between Western Australia and United Kingdom, Psychotherapy Theory and Practice 2006;22:317-28.

5. Dunn LB, Iglewicz A, Moutier C. A Conceptual Model of Medical Student Well-Being: Promoting Resilience and Preventing Burnout. Acad Psychiatry 2008;2:44-53.

6. Galbraith ND, Brown KE. Assessing Intervention Effectiveness for Reducing Stress in Student Nurses: Quantitative Systematic Review. J Adv Nurs 2011; 67:709-21.

7. Henning K, Ey S, Shaw D. Perfectionism, the Impostor Phenomenon and Psychological Adjustement in Medical, Dental, Nursing and Pharmacy. Med Educ 1998;32:456-64.
8. Pfeifer TA, Kranz PL, Scoggin AE. Perceived Stress in Occupational Therapy Students. Occup Ther Int 2008;15:221-31.

9. Roberts L W, Warner T D, Lyketsos C, Frank E, Ganzini L. Perceptions of Academic Vulnerability Associated with Personal Illness: A Study of 1,027 Students at Nine Medical Schools. Compr Psychiatry 2001;42:1-15.

10. Harrison E. Faculty Perceptions of Academic Advising. "I Don't Get No Respect". Nursing Education Research 2009;30:229-33.

11. Levy BD, Katz JT, Wold MA, Sillman JS, Handin RI, Dzau VJ. An Initiative in Mentoring to Promote Residents' and Faculty Members' Careers. Acad Med 2004;79:845-50.

12. Sambujak D, Straus SE, Marusie A. Mentoring in Academic Medicine. A Systematic Review. JAMA 2006;296:1103-15.

13. Lemire S, Snyder C, Heuertz L. Preliminary Report. UW Academic Advising Self-Study 2004-2005. USA: Office of Educational Assessment. Improving Learning Through Assessment. 2005.

14. Coll JE, Draves, P. Traditionnal Age Students: Worldviews and Satisfaction with Advising: A Homogeneous Study of Students and Advisors. The College Student Affairs Journal 2009;28:215-21.

15. Hale MD, Graham DL, Johnson DM. Are Students More Satisfied with Academic Advising when There is Congruence between Current and Preferred Advising Styles? College Student Journal 2009;32: 313-24.

16. Dyrbye LN, Power DV, Massie FS, Eacker A, Harper W, Thomas MR, et al. Factors Associated with Resilience to and Recovery from Burnout: A Prospective, Multi-Institutional Study of US Medical Students. Med Educ 2010;44:1016-26.

17. Wrigh SM, Kern DE, Koloder K, Howard DM, Brancati FL. Attributes of Excellent AttendingPhysician Role Models. New Engl J Med 1998;339: 1986-93.

18. Kjeldstadli K, Tyssen R, Finset A, Hem E, Gude T, Gronvold NT, et al. Life Satisfaction and Resilience in Medical School - A Six-Year Longitudinal, Nationwide and Comparative Study, BMC Med Educ 2006;6:48.

Correspondance et offprints: Sophie Laflamme, Faculté de médecine et des sciences de la santé, Université de Sherbrooke, Québec, Canada.

Mailto : Sophie.Laflamme@USherbrooke.ca 